\title{
BMJ Open Efficacy and safety of hyperbaric oxygen therapy for moderate-to-severe ulcerative colitis: a protocol for a systematic review and meta-analysis
}

\author{
Lihong Luo (1) , Lei Qing, Chengjiao Yao, Dongying Liu, Yilin Li, Tinglin Li, \\ Peimin Feng
}

To cite: Luo L, Qing L, Yao C, et al. Efficacy and safety of hyperbaric oxygen therapy for moderate-to-severe ulcerative colitis: a protocol for a systematic review and meta-analysis. BMJ Open 2021;11:e047543. doi:10.1136/ bmjopen-2020-047543

- Prepublication history for this paper is available online. To view these files, please visit the journal online (http://dx.doi org/10.1136/bmjopen-2020047543).

Received 03 December 2020 Accepted 27 May 2021

Check for updates

(c) Author(s) (or their employer(s)) 2021. Re-use permitted under CC BY-NC. No commercial re-use. See rights and permissions. Published by BMJ.

Hospital of Chengdu University of Traditional Chinese Medicine, Chengdu, Sichuan Province, China

Correspondence to Professor Peimin Feng; fpmvv@126.com

\section{ABSTRACT}

Introduction Ulcerative colitis (UC) is a type of inflammatory bowel disease, and $62 \%$ of patients with UC felt that it is difficult for them to live a normal life. Furthermore, some researches have shown that about $15 \%$ of patients with UC undergo at least one extreme clinical course in their lifetime, and $10 \%-30 \%$ of patients with UC oblige colectomy. Although many investigations have demonstrated that $\mathrm{HBO}_{2}$ has a beneficial impact on UC treatment, a systematic review and meta-analysis are unavailable. Therefore, a meta-analysis is essential to assess the efficacy and safety of $\mathrm{HBO}_{2}$ in treating UC. Methods and analysis A systematic search plan will be performed in the following seven databases with a restriction of time from inception to September 2020 to filter the eligible studies: PubMed, Web of Science, Embase, Cochrane Library, China National Knowledge Infrastructure, Chinese Scientific Journal Database (VIP) and Chinese Biomedical Database WanFang. Other related resources will be also searched. Two independent reviewers will choose eligible researches and extract data. The risk of bias will be evaluated based on Cochrane Collaboration's Risk of Bias tool and Newcastle-Ottawa Scale. Eventually, a systematic review and meta-analysis will be performed via the Review Manager V.5.3 statistical software and STATA V.14.0 software.

Ethics and dissemination This study will not involve the individual patient and any ethical problems since its outcomes are based on published data. Therefore, no ethical review and approval are required. We plan to publish the study in a peer-reviewed journal.

PROSPERO registration number CRD42020210244.

\section{INTRODUCTION}

\section{Description of the condition}

Ulcerative colitis (UC) is a type of inflammatory bowel disease (IBD) characterised by idiopathic, diffuse inflammation of colonic mucosa. ${ }^{1}$ The peak age for UC occurrence is $30-40$ years, without sex difference. Some researches have indicated that a second peak onset occurs at 60-70years old, but this statement needs to be further demonstrated. ${ }^{2}$ Although the aetiology and pathogenesis of
Strengths and limitations of this study

- This systematic review and meta-analyses will be the latest report to answer the clinical question of whether $\mathrm{HBO}_{2}$ should be promoted and applied in patients with moderate-to-severe ulcerative colitis.

- Screening of search citations, full-text screening, data extraction, risk of bias and quality assessment will be completed independently by at least two reviewers and a third researcher as an arbitrator.

- However, since $\mathrm{HBO}_{2}$ protocol types used in various studies may be different, the research conclusions may be biased to some extent.

- Studies published in languages other than English or Chinese may be omitted due to language limitations, which may lead to language bias.

UC remain unknown, it has been established that several factors contribute to UC development. These factors include environmental factors (changes in the intestinal microbiome resulting from certain medications, diet and smoking), genetic vulnerability, aberrant host immune responses and disturbance of intestinal barrier equilibrium. ${ }^{34}$ The typical gastrointestinal disorders of UC mainly include diarrhoea, bloody stool, abdominal pain and rectal urgency. In addition to the above symptoms, some patients with UC may present other multiple extraintestinal manifestations, such as oral ulcer, skin disorders, osteoporosis, eye inflammation and arthritis. ${ }^{5}$ Recurrent episodes of colonic inflammation seriously affect lives and work of patients with $\mathrm{UC}$, as well as their psychological well-being, and may also raise the risk of colorectal cancer. Among patients with UC, $62 \%$ experienced a challenging normal life, ${ }^{6} 15 \%$ underwent at least one extreme clinical course in their lifetime and 10\%-30\% of them obliged colectomy. ${ }^{78} \mathrm{UC}$ incidence varies significantly 
between different countries and regions. Specifically, the highest UC incidence was in Europe $(0.505 \%$ in Norway) and North America $(0.286 \%$ in the USA), while UC has a low incidence in developing countries and regions. However, due to the industrialisation development, UC incidence in Asia, South America and Africa has gradually increased over the last decades. ${ }^{9}$ According to recent epidemiological data, UC has become a global disease, imposing a notable socioeconomic burden on the healthcare system. ${ }^{10}$ Burisch et al assessed the healthcare expenditures of UC in the first 5 years after being diagnosed in Europe using Epi-IBD cohort and determined that the mean annual healthcare costs for one patient with UC per year were $2088 €$ during follow-up. ${ }^{11}$

At present, the recommended treatment goals in UC are to induce and maintain clinical remission, which means bloody stool absence and stool frequency normalisation, and endoscopic remission, which is defined as a Mayo endoscopic subscore (MES) of 0 or $1 .{ }^{12} 13$ The main conventional medications for UC include aminosalicylates, corticosteroids and immunomodulators such as azathioprine and methotrexate. ${ }^{14}$ Nevertheless, approximately $20 \%-40 \%$ of patients with UC poorly respond to these drugs. ${ }^{15}$ Since the late 1990s, when biologic agents (such as anti-tumor necrosis factor, cytokine inhibitors) were approved, the treatment and management of UC have advanced significantly. ${ }^{16}$ An investigation has shown that colectomy rates decreased as the utilisation of biological agents increased. ${ }^{17}$ However, many shortcomings with biologic therapies are present, such as low compliance and high expenditure. Wentworth et al assessed vedolizumab in patients with IBD, with an overall adherence rate of $83 \% .{ }^{18}$ As a new therapy, doctors and patients need to be aware of the associated risks, such as malignancy, infections, infusion/injection site reactions, and so on. ${ }^{19}$ In addition, $30 \%$ of patients with UC do not respond to anti-TNF, and about a third eventually lose response to the drug. ${ }^{20}$ Therefore, there is an urgent need for safer and more efficient non-drug treatment alternatives for UC, such as hyperbaric oxygen therapy and faecal transplant.

\section{Description of the intervention}

The application of hyperbaric air dates back to 1667 . Hyperbaric oxygen therapy $\left(\mathrm{HBO}_{2}\right)$ is defined as breathing close to $100 \%$ oxygen in a hyperbaric oxygen chamber, where the pressure exceeds 1.4 absolute atmospheres (ATA). ${ }^{21} \mathrm{HBO}_{2}$ therapy is performed in 2-3 absolute atmospheric pressure chambers 2-3 times daily. The length of treatment varies according to distinct indications but is usually between 1.5 and 2 hours. ${ }^{22}$ After more than 300 years of development, $\mathrm{HBO}_{2}$ therapy has been demonstrated to be safe and effective in treating various diseases, with few side effects. According to Undersea and Hyperbaric Medical Society (UHMS), ${ }^{23} \mathrm{HBO}_{2}$ has been approved for use in recognised indications, such as air or gas embolism, decompression sickness, severe anaemia, intracranial abscess and carbon monoxide poisoning. In addition, without UHMS approval, $\mathrm{HBO}_{2}$ has some potential indications, including UC, Raynaud syndrome, otitis externa, and so on. ${ }^{24} \mathrm{~A}$ phase IIB randomised trial revealed that, after receiving $\mathrm{HBO}_{2}, 85 \%$ of patients hospitalised for acute flares could avert second-line therapy (colectomy and biological agent). Furthermore, approximately $70 \%$ of patients can achieve remission or near-complete remission of rectal bleeding. ${ }^{25}$ As a result, $\mathrm{HBO}_{2}$ improved survival in patients with moderate-tosevere UC.

\section{How the intervention might work}

$\mathrm{HBO}_{2}$ involves breathing $100 \%$ oxygen under increased atmospheric pressure, which significantly increases the oxygen levels in plasma and tissues to promote wound healing. ${ }^{22}$ Although high oxygen levels produced by hyperbaric oxygen are only maintained when the patient is in the hyperbaric oxygen chamber, and for a short time afterward, $\mathrm{HBO}_{2}$ can also produce various biochemical effects, including (1) inhibition of neutrophils' adhesion and production of proinflammatory cytokines (interleukin-1 (IL-1), IL-6 and TNF-a), (2) upregulation of hypoxia response pathway (HIF-1 $\alpha, \mathrm{HO}-1$ ), (3) changes in host-microbiome metabolism and (4) increased growth factor synthesis and migration. ${ }^{26-31}$

\section{OBJECTIVES}

Some studies have demonstrated that $\mathrm{HBO}_{2}$ can relieve a range of symptoms of patients who suffer from moderateto-severe UC. On the contrary, Pagoldh et al conducted a prospective randomised study and indicated that $\mathrm{HBO}_{2}$ is ineffective in treating UC. ${ }^{32}$ Dulai et al conducted a systematic review of safety and effectiveness of hyperbaric oxygen in treating IBD (including Crohn's disease and UC) in 2014, and they concluded that hyperbaric oxygen is a relatively safe and potentially effective option for IBD treatment. ${ }^{33}$ After careful assessment of this work, we found that the patients included in this systematic review had Crohn's disease and UC, and this systematic review did not separately investigate the safety of $\mathrm{HBO}_{2}$ for UC. Therefore, we believe that this conclusion has limited guidance for gastroenterologists in treating UC. In addition, we also noted that there were some latest studies on hyperbaric oxygen therapy for patients with UC after 2014. Consequently, we intend to perform a systematic review and meta-analysis to quantify the safety and efficacy of $\mathrm{HBO}_{2}$ for UC. In summary, our study results will provide reliable reference information for patients and physicians when selecting treatment options for UC.

\section{METHODS AND ANALYSIS}

\section{Study design}

The design of this protocol strictly follows the guidelines and recommendations of the systematic review and metaanalysis priority report item (Preferred Reporting Items for Systematic Review and Meta-Analysis Protocols). ${ }^{34}$ The methodology is preregistered on the International 
Prospective Register of Systematic Reviews (PROSPERO) with a registration ID of CRD42020210244.

\section{Inclusion/exclusion criteria for study selection \\ Types of studies}

Randomised controlled trials (RCTs) and observational studies (cohort and case-control) will be included. Articles including experimental animals, narrative reviews, cross-sectional studies, expert opinions and editorials will be excluded. The language of the studies has a restriction of English or Chinese.

\section{Types of participants}

Inclusion criteria: studies of adult patients who suffer from moderate-to-severe UC will be considered. In other words, those patients with a full Mayo score $\geq 6$ and MES of 2 or 3 will be included, irrespective of gender, race and education level.

Exclusion criteria: pregnant women and those patients who have a clear contraindication to $\mathrm{HBO}_{2}$ therapy, for example, cataract, age-related macular degeneration or pneumothorax will be excluded. ${ }^{35}$ In addition, patients who need urgent colectomy due to severe toxic megacolon will be excluded.

\section{Types of interventions/controls}

All studies evaluating hyperbaric oxygen therapy for moderate-to-severe UC will be included. Interventions mainly include the following two types: (1) $\mathrm{HBO}_{2}$ therapy alone, without limiting the depth, duration and frequency of hyperbaric oxygen; (2) $\mathrm{HBO}_{2}$ therapy combined with the main conventional medications for UC, regardless of dose and route of administration, such as aminosalicylates, corticosteroids, immunomodulators and biological agents. If the intervention is only involved in $\mathrm{HBO}_{2}$ therapy, the control group can select sham $\mathrm{HBO}_{2}$. Otherwise, the experimental and control groups should use the same conventional drug treatment, except for $\mathrm{HBO}_{2}$ therapy.

\section{Types of outcome measures \\ Primary outcomes}

Since our study aims to systematically assess $\mathrm{HBO}_{2}$ on moderate-to-severe UC, we will select the Mayo score and the MES as the primary outcomes, which can reflect the activity of UC to a certain extent. In addition to the above scores, faecal calprotectin and serum inflammatory factors were found to be reliable indicator of UC activity. ${ }^{36}$

\section{Secondary outcomes}

The secondary outcomes mainly include safety, prevention of colectomy and clinical response from patients. The safety of $\mathrm{HBO}_{2}$ is chiefly measured by the incidence of adverse effects and serious adverse events.

\section{Patient and public involvement}

Patients and/or public were not involved in design, or conduct, or reporting or dissemination plans for this research.

\section{Box 1 Search strategy in PubMed database.}

\section{Search items}

1. Ulcerative colitis.MeSh.

2. Colitis, Ulcerative.ti.ab.

3. UC.ti.ab.

4. IBD.ti.ab.

5. 1 or $2-4$

6. Hyperbaric oxygen.MeSh.

7. Hyperbaric Oxygenations. ti.ab.

8. Oxygenations, Hyperbaric. ti.ab.

9. Hyperbaric Oxygen Therapy. ti.ab.

10. Hyperbaric Oxygen Therapies. ti.ab.

11. Oxygenation, Hyperbaric. ti.ab.

12. Oxygen Therapy, Hyperbaric. ti.ab.

13. Therapies, Hyperbaric Oxygen. ti.ab.

14. 6 or $7-13$

15. 5 and 14

\section{Search resources}

\section{Electronic searches}

A systematic search plan will be performed in the following seven databases with a restriction of time from inception to September 2020 to filter the eligible studies: PubMed, Web of Science, Embase, Cochrane Library, China National Knowledge Infrastructure, Chinese Scientific Journal Database (VIP) and Chinese Biomedical Database WanFang.

\section{Clinical trial registers}

The following two clinical trials registry platforms were searched: (1) the US National Institutes of Health Ongoing Trials Register and () the International Clinical Trials Registry Platform.

\section{Other sources}

We will search other related resources as far as possible by browsing the reference of eligible studies and the other related grey literature (conference, papers, and journal articles).

\section{Search strategies}

We will use a combination of subject terms and accessible text terms for retrieval. Indeed, there is a little difference in retrieval strategies in different databases. Therefore, we considered the specific search strategy in PubMed as a typical example, and the specific steps of the retrieval are shown in Box 1.

\section{Data collection and analysis \\ Selection of studies}

First, two independent reviewers (LL and CY) will use the EndNote X9 software to read the titles, keywords and abstracts of all obtained studies. Subsequently, the eligibility will be confirmed after screening the full text of potentially eligible studies. Any disagreements will be resolved through negotiation and consensus. Further controversy will be arbitrated by a third reviewer (LQ) if necessary. In summary, the entire selection process will be completed 


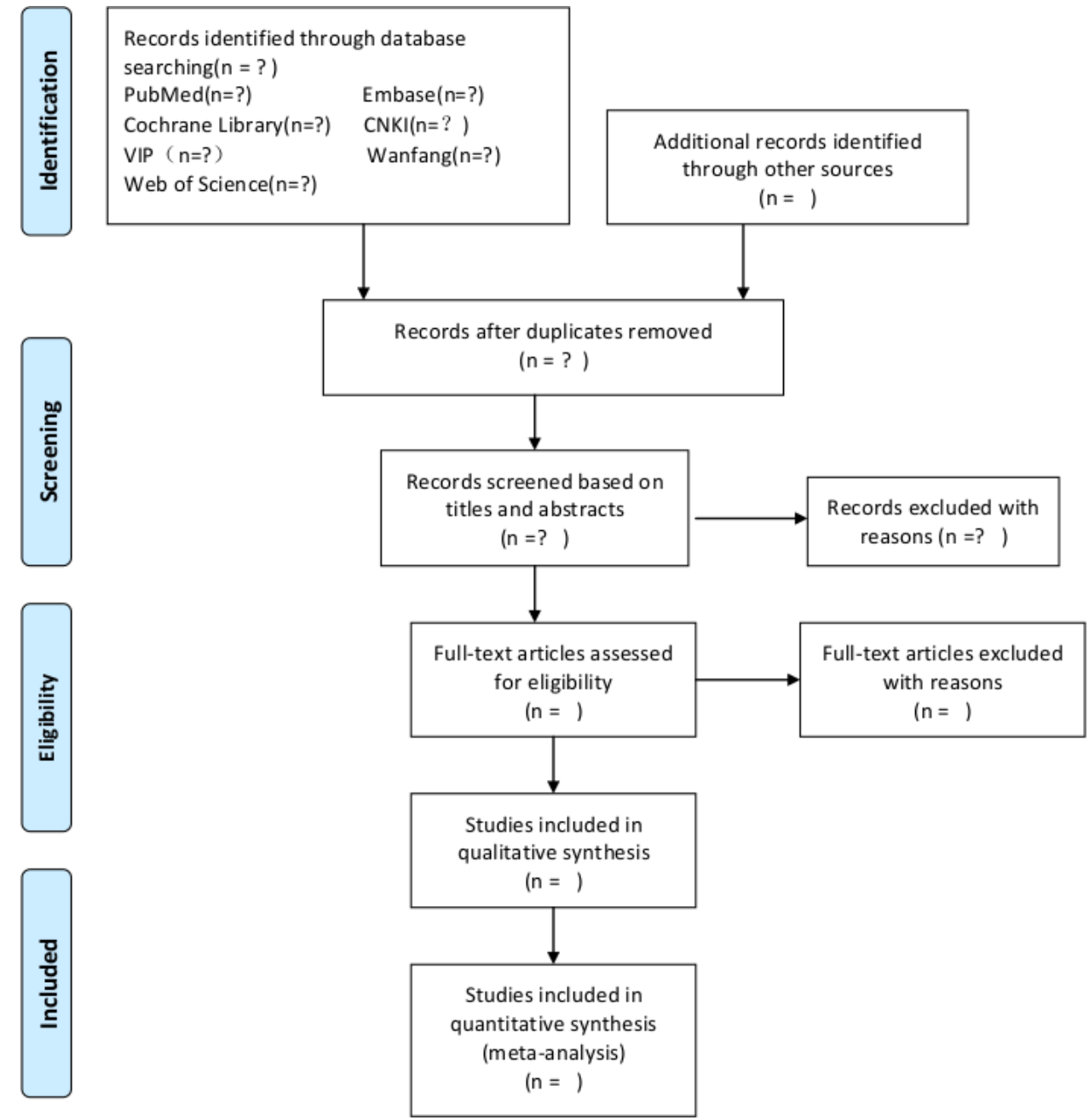

Figure 1 Flow chart diagram presenting the selection process for the studies. This figure shows the identification, screening, eligibility and included when searching articles. CNKI, China National Knowledge Infrastructure.

independently by at least two authors, and the exclusion reasons for each excluded study will be noted. Figure 1 demonstrates the steps in the study screening process.

\section{Data extraction and management}

Two independent researchers (TL and LQ) will apply a predesigned data collection form to extract data from included references. If there are any disagreements, the third reviewer (DL) will be consulted. The extracted data items mainly contain the following four parts:

1. Basic information of studies (year of publication, the first author, country, sample size and follow-up time)

2. Participants (gender, age, area, duration and degree of UC, some blood biomarkers, MES and Ulcerative Colitis Endoscopic Index of Severity).

3. Treatment (interventions, controls, type of $\mathrm{HBO}_{2}$ chamber, $\mathrm{HBO}_{2}$ protocol (depth, duration, prophylactic air breaks, frequency and treatment duration)).

4. Outcomes (Mayo score, the MES, faecal calprotectin, and adverse events).

\section{Assessment of risk of bias}

Two independent reviewers (YL and DL) evaluated the risk of bias of RCTs using the Cochrane Collaboration's
Risk of Bias Tool. The assessed domains consist of the following items: random sequence generation, allocation concealment, blinding of participants and personnel, blinding of outcome assessment, incomplete outcome data, selective reporting and other biases. We will confirm each item from 3 levels of 'high risk', 'low risk' and 'unclear'. Any discrepancies will be arbitrated by negotiation with a fourth reviewer $(\mathrm{PF})$. As for the cohort studies and case-control studies, we intend to use the Newcastle-Ottawa Scale (NOS) to assess the risk of bias. NOS consist of the following items: selection, exposure and comparability.

\section{Assessment of publication biases}

If more than 10 studies are included, the publication bias will be conducted through a funnel plot. The funnel plot method can qualitatively identify publication bias, while Begg's rank correlation test and Egger's linear regression test can quantitatively judge whether there is publication bias by examining the $p$ value. We will use Begg's rank correlation test and Egger's linear regression test to examine the symmetry of funnel plots if sufficient studies are available. In the case of poor symmetry of the funnel 
plot, the trim and fill method will also be performed. Since the test power of the above methods is closely related to the number of included studies, we will make a careful selection based on the number of included studies in our specific analysis.

\section{Measures of treatment effect}

According to different data types, we will apply various measures to assess the effect size of each included study. For continuous outcomes (Mayo score, the MES, faecal calprotectin and serum inflammatory factors), the weighted mean difference or the standard mean difference will be calculated for analysis. Dichotomous outcomes (colectomy, adverse events, serious adverse events and clinical response about remission of symptoms from patients) data will be expressed as the risk ratio with 95\% CIs.

\section{Dealing with missing data}

We will contact the corresponding authors via email as far as possible to obtain the missing data. In case of failure, we will eliminate this study from the analysis and give a rational explanation.

\section{Assessment of heterogeneity}

We will mainly adopt the following methods to evaluate the heterogeneity of the included studies: $\mathrm{I}^{2}$ and the forest plot. This operation will be carried out using the Review Manager (V.5.3.5). Statistical heterogeneity among studies will be evaluated with $\mathrm{I}^{2}$ statistic, with $\mathrm{I}^{2}<25 \%$ indicating no heterogeneity, with $\mathrm{I}^{2}<50 \%$ expressing low heterogeneity, $\mathrm{I}^{2}<75 \%$ indicating moderate heterogeneity, and with $\mathrm{I}^{2} \geq 75 \%$ expressing high heterogeneity. ${ }^{37}$

\section{Data synthesis}

We will use Review Manager V.5.3 software provided by the Cochrane Collaboration to implement the statistical analyses. If necessary, STATA software V.14.0 (STATA Corporation) will also be used for statistical analyses. If the eligible studies are sufficiently homogeneous, data from all studies will be pooled for a meta-analysis. If the included studies exhibit low heterogeneity $\left(\mathrm{I}^{2}<50 \%\right)$, we will conduct the statistical combination via a fixed-effects model. On the contrary, we will choose the randomeffects model. Subgroup analysis will also be carried out to explore potential sources of heterogeneity, while sensitivity analysis will be performed to evaluate the robustness and reliability of each outcome. ${ }^{38}$ We will perform descriptive summaries in the case of a meta-analysis without feasibility due to significant statistical heterogeneity.

\section{Subgroup analysis}

If substantial heterogeneity exists between studies, a subgroup analysis will be performed to determine the cause of heterogeneity. Currently, we plan to conduct this analysis according to characteristics of participants (age, gender, race or stage of UC), types of $\mathrm{HBO}_{2}$ protocol (depth, duration, break, frequency and the course of treatment), type of standard medical therapy (immunosuppressive drugs, 5-aminosalicylic acid or steroids). In addition, we also intend to conduct subgroup analysis based on the level of evidence and risk of bias in the included literature, which can more accurately and comprehensively explore heterogeneity sources. However, during actual implementation, the subgroup analysis will not be restricted to the planned subgroup and incorporate some adjustments based on the extracted data. To further improve the subgroup analysis reliability, it will be evaluated based on the guidance for credible subgroup analysis. If the data of the included studies are available and sufficient, a meta-regression will be performed to determine heterogeneity.

\section{Sensitivity analysis}

To evaluate the robustness and reliability of each outcome, a sensitivity analysis will be carried out. We plan to repeat the meta-analysis based on the remaining data after removing each study one by one and confirm whether the pooled results are robust and reliable via comparing the before and after results.

\section{Evaluating the evidence}

Two reviewers (LL and LQ) will assess the quality of evidence according to the Grading of Recommendations Assessment, Development and Evaluation, which classifies the evidence into four levels: very low, low, moderate and high levels. ${ }^{39}$

\section{Ethics and dissemination}

This study will not involve the individual patient and any ethical problems since its outcomes are based on published data. Therefore, no ethical review and approval are required in this study. We plan to publish the study in a peer-reviewed journal.

Contributors $L L$ and $L Q$ are joint first authors. $L L$ and $Q L$ initiated the idea and led the development of this protocol. CY, DL, YL, TL and PF were involved in the planning and design process of this protocol. $\mathrm{LL}$ and $\mathrm{CY}$ conducted the selection of studies. Data extraction will be performed by TL and QL. The assessment of the risk of bias will be carried out by $\mathrm{DL}$ and YL. Any discrepancies will be resolved by discussion with a third reviewer PF. PF will monitor each procedure of the review. All authors read and approved the final manuscript.

Funding This study is funded by the National Key R\&D Program of China (2018YFC1705403).

Competing interests None declared.

Patient and public involvement Patients and/or the public were not involved in the design, or conduct, or reporting or dissemination plans of this research.

Patient consent for publication Not required.

Provenance and peer review Not commissioned; externally peer reviewed.

Open access This is an open access article distributed in accordance with the Creative Commons Attribution Non Commercial (CC BY-NC 4.0) license, which permits others to distribute, remix, adapt, build upon this work noncommercially, and license their derivative works on different terms, provided the original work is properly cited, appropriate credit is given, any changes made indicated, and the use is non-commercial. See: http://creativecommons.org/ licenses/by-nc/4.0/.

ORCID iD

Lihong Luo http://orcid.org/0000-0001-9055-1137 


\section{REFERENCES}

1 Ordás I, Eckmann L, Talamini M, et al. Ulcerative colitis. Lancet 2012;380:1606-19.

2 Cosnes J, Gower-Rousseau C, Seksik P, et al. Epidemiology and natural history of inflammatory bowel diseases. Gastroenterology 2011;140:1785-94.

3 Ramos GP, Papadakis KA. Mechanisms of disease: inflammatory bowel diseases. Mayo Clin Proc 2019;94:155-65.

4 Sartor RB. Mechanisms of disease: pathogenesis of Crohn's disease and ulcerative colitis. Nat Clin Pract Gastroenterol Hepatol 2006;3:390-407.

5 Stein P. Ulcerative colitis--diagnosis and surgical treatment. Aorn J 2004;80:242-62.

6 Rubin DT, Siegel CA, Kane SV, et al. Impact of ulcerative colitis from patients' and physicians' perspectives: results from the Uc: normal survey. Inflamm Bowel Dis 2009;15:581-8.

7 Aratari A, Papi C, Clemente V, et al. Colectomy rate in acute severe ulcerative colitis in the infliximab era. Dig Liver Dis 2008;40:821-6.

8 Turner D, Walsh CM, Steinhart $\mathrm{AH}$, et al. Response to corticosteroids in severe ulcerative colitis: a systematic review of the literature and a meta-regression. Clin Gastroenterol Hepatol 2007;5:103-10.

9 SC N, Shi HY, Hamidi N. Worldwide incidence and prevalence of inflammatory bowel disease in the 21st century: a systematic review of population-based studies. Lancet 2018;390:2769-78.

10 Pillai N, Dusheiko M, Maillard MH, et al. The evolution of health care utilisation and costs for inflammatory bowel disease over ten years. J Crohns Colitis 2019;13:744-54.

11 Burisch J, Vardi H, Schwartz D, et al. Health-Care costs of inflammatory bowel disease in a pan-European, community-based, inception cohort during 5 years of follow-up: a population-based study. Lancet Gastroenterol Hepatol 2020;5:454-64.

12 Jairath V, Khanna R, Zou GY, et al. Development of interim patientreported outcome measures for the assessment of ulcerative colitis disease activity in clinical trials. Aliment Pharmacol Ther 2015;42:1200-10.

13 Peyrin-Biroulet L, Sandborn W, Sands BE, et al. Selecting therapeutic targets in inflammatory bowel disease (STRIDE): determining therapeutic goals for Treat-to-Target. Am J Gastroenterol 2015;110:1324-38

14 Stange EF, Travis SPL. The European consensus on ulcerative colitis: new horizons? Gut 2008;57:1029-31.

15 Park SC, Jeen YT. Current and emerging biologics for ulcerative colitis. Gut Liver 2015;9:18-27.

16 Koliani-Pace JL, Haron AM, Zisman-Ilani Y, et al. Patients' perceive biologics to be Riskier and more dreadful than other IBD medications. Inflamm Bowel Dis 2020;26:141-6.

17 Barnes EL, Jiang Y, Kappelman MD, et al. Decreasing colectomy rate for ulcerative colitis in the United States between 2007 and 2016: a time trend analysis. Inflamm Bowel Dis 2020;26:1225-31.

18 Wentworth BJ, Buerlein RCD, Tuskey AG, et al. Nonadherence to biologic therapies in inflammatory bowel disease. Inflamm Bowel Dis 2018;24:2053-61.

19 Bonovas S, Fiorino G, Allocca M, et al. Biologic therapies and risk of infection and malignancy in patients with inflammatory bowel disease: a systematic review and network meta-analysis. Clin Gastroenterol Hepatol 2016;14:1385-97.
20 Panés J, Alfaro I. New treatment strategies for ulcerative colitis. Expert Rev Clin Immunol 2017;13:963-73.

21 Harlan NP, Ptak JA, Rees JR, et al. Development of an international, multicenter, hyperbaric oxygen treatment registry and research Consortium: protocol for outcome data collection and analysis. JMIR Res Protoc 2020;9:e18857.

22 Thom SR. Hyperbaric oxygen: its mechanisms and efficacy. Plast Reconstr Surg 2011;127 Suppl 1:131S-41.

23 Undersea and Hyperbaric Medical SocietyMoon RE. Hyperbaric oxygen therapy indications. 14th edn. North Palm Beach, FL: Best Publishing, 2019.

24 Thom SR. Oxidative stress is fundamental to hyperbaric oxygen therapy. J Appl Physiol 2009;106:988-95.

25 Dulai PS, Raffals LE, Hudesman D. A phase $2 B$ randomised trial of hyperbaric oxygen therapy for ulcerative colitis patients hospitalised for moderate to severe flares. Alimentary Pharmacology and Therapeutics;2020.

26 Neish AS, Jones RM. Redox signaling mediates symbiosis between the gut microbiota and the intestine. Gut Microbes 2014;5:250-3.

27 Rothfuss A, Radermacher P, Speit G. Involvement of heme oxygenase-1 (HO-1) in the adaptive protection of human lymphocytes after hyperbaric oxygen (HBO) treatment. Carcinogenesis 2001;22:1979-85.

28 Thom SR. Effects of hyperoxia on neutrophil adhesion. Undersea Hyperb Med 2004;31:123-31.

29 Weisz G, Lavy A, Adir Y, et al. Modification of in vivo and in vitro TNF-alpha, IL-1, and IL-6 secretion by circulating monocytes during hyperbaric oxygen treatment in patients with perianal Crohn's disease. J Clin Immunol 1997;17:154-9.

30 Yamashita M, Yamashita M. Hyperbaric oxygen treatment attenuates cytokine induction after massive hemorrhage. Am J Physiol Endocrinol Metab 2000;278:E811-6.

31 Peng Z, Ren P, Kang Z, et al. Up-Regulated HIF-1alpha is involved in the hypoxic tolerance induced by hyperbaric oxygen preconditioning Brain Res 2008;1212:71-8.

32 Pagoldh M, Hultgren E, Arnell P, et al. Hyperbaric oxygen therapy does not improve the effects of standardized treatment in a severe attack of ulcerative colitis: a prospective randomized study. Scand J Gastroenterol 2013;48:1033-40.

33 Dulai PS, Gleeson MW, Taylor D, et al. Systematic review: the safety and efficacy of hyperbaric oxygen therapy for inflammatory bowel disease. Aliment Pharmacol Ther 2014;39:1266-75.

34 Moher D, Shamseer L, Clarke M, et al. Preferred reporting items for systematic review and meta-analysis protocols (PRISMA-P) 2015 statement. Syst Rev 2015;4:1.

35 McMonnies CW. Hyperbaric oxygen therapy and the possibility of ocular complications or contraindications. Clin Exp Optom 2015:98:122-5.

36 Buisson A, Mak WY, Andersen MJ, et al. Faecal calprotectin is a very reliable tool to predict and monitor the risk of relapse after therapeutic De-escalation in patients with inflammatory bowe diseases. J Crohns Colitis 2019;13:1012-24.

37 Higgins JPT, Thompson SG. Quantifying heterogeneity in a metaanalysis. Stat Med 2002;21:1539-58.

38 Higgins JPT, Thompson SG, Deeks JJ, et al. Measuring inconsistency in meta-analyses. BMJ 2003;327:557-60.

39 Balshem H, Helfand M, Schünemann HJ, et al. Grade guidelines: 3. rating the quality of evidence. J Clin Epidemiol 2011;64:401-6. 